PROCEEDINGS OF THE

AMERICAN MATHEMATICAL SOCIETY

Volume 130, Number 12, Pages 3525-3535

S 0002-9939(02)06617-0

Article electronically published on May 15, 2002

\title{
ALGEBRAIC OBSTRUCTIONS AND A COMPLETE SOLUTION OF A RATIONAL RETRACTION PROBLEM
}

\author{
RICCARDO GHILONI
}

(Communicated by Paul Goerss)

\begin{abstract}
For each compact smooth manifold $W$ containing at least two points we prove the existence of a compact nonsingular algebraic set $Z$ and a smooth map $g: Z \longrightarrow W$ such that, for every rational diffeomorphism $r: Z^{\prime} \longrightarrow Z$ and for every diffeomorphism $s: W^{\prime} \longrightarrow W$ where $Z^{\prime}$ and $W^{\prime}$ are compact nonsingular algebraic sets, we may fix a neighborhood $\mathcal{U}$ of $s^{-1} \circ g \circ r$ in $C^{\infty}\left(Z^{\prime}, W^{\prime}\right)$ which does not contain any regular rational map. Furthermore $s^{-1} \circ g \circ r$ is not homotopic to any regular rational map. Bearing in mind the case in which $W$ is a compact nonsingular algebraic set with totally algebraic homology, the previous result establishes a clear distinction between the property of a smooth map $f$ to represent an algebraic unoriented bordism class and the property of $f$ to be homotopic to a regular rational map. Furthermore we have: every compact Nash submanifold of $\mathbb{R}^{n}$ containing at least two points has not any tubular neighborhood with rational retraction.
\end{abstract}

\section{INTRODUCTION}

This paper deals with algebraic obstructions. First, it is necessary to recall part of the classical problem of making smooth objects algebraic.

Let $M$ be a smooth manifold. A nonsingular real algebraic set $V$ diffeomorphic to $M$ is called an algebraic model of $M$. In [28. Tognoli proved that any compact smooth manifold has an algebraic model. By virtue of this result it is natural to wonder if a geometric object of $M$ as a homology class $\alpha \in H_{*}(M, \mathbb{Z} / 2 \mathbb{Z})$ can be realized algebraically in some algebraic model of $M$. Such a question may be subdivided in two complementary problems: 1-total obstruction problem) find smooth compact manifolds $M$ and homology classes $\alpha \in H_{*}(M, \mathbb{Z} / 2 \mathbb{Z})$ such that, in every algebraic model $M^{\prime}$ of $M, \alpha$ is not represented by any real algebraic subset of $M^{\prime}$; 2-constructive problem) given a subgroup $G$ of $H_{k}(M, \mathbb{Z} / 2 \mathbb{Z})$ for some integer $k$, find an algebraic model $M^{\prime}$ such that $G$ corresponds to the subgroup $H_{k}^{a l g}\left(M^{\prime}, \mathbb{Z} / 2 \mathbb{Z}\right)$ of $H_{k}\left(M^{\prime}, \mathbb{Z} / 2 \mathbb{Z}\right)$ generated by the homology classes represented by all $k$-dimensional real algebraic subsets of $M^{\prime}$. The latter problem has been investigated by Bochnak and Kucharz in [1] and [12 (see also section 11.3 of [8). With regard to the first problem Benedetti and Dedò [5] proved the following theorem which, in the final analysis, even today remains the unique result regarding algebraic obstructions up to homeomorphism.

Received by the editors August 1, 2001.

2000 Mathematics Subject Classification. Primary 14P05; Secondary 14P20, 14P25.

Key words and phrases. Algebraic obstructions, regular rational retractions.

(C)2002 American Mathematical Society 
Benedetti-Dedò theorem. For each $d \geq 11$ there exists a d-dimensional compact connected smooth manifold $M$ and a homology class $\alpha \in H_{d-2}(M, \mathbb{Z} / 2 \mathbb{Z})$ such that, for every homeomorphism $h: M \longrightarrow M^{\prime}$ where $M^{\prime}$ is an algebraic manifold, the homology class $h_{*}(\alpha)$ of $M^{\prime}$ is not contained in $H_{d-2}^{\text {alg }}\left(M^{\prime}, \mathbb{Z} / 2 \mathbb{Z}\right)$.

Later, Teichner 26] showed that such a result is true for each $d \geq 6$ which is the best possible (see Theorem 11.3.12 of [8]). The Benedetti-Dedò-Teichner theorem has the following corollary (see page 144 of [5]).

Corollary. For each $d \geq 6$ there exist a d-dimensional compact connected smooth manifold $M, a(d-2)$-dimensional compact connected smooth manifold $N$ and a smooth map $f: N \longrightarrow M$ such that, for every algebraic model $N^{\prime}$ of $N$ and $M^{\prime}$ of $M$, the map $f$ viewed as a smooth map from $N^{\prime}$ to $M^{\prime}$ is not approximable by regular rational maps.

In this note, we make progress in the direction of the above obstruction corollary. Before stating our main theorem (Theorem 1) and one of its consequences (Theorem 2), we shall specify the meaning of some notions mentioned above and shall give other definitions.

Let $V$ be a subset of $\mathbb{R}^{n}$, let $W$ be a subset of $\mathbb{R}^{m}$ and let $f: V \longrightarrow W$ be a map. $f$ is a regular rational map if, for each $x \in V$, there are a Zariski neighborhood $U$ of $x$ in $\mathbb{R}^{n}$, a polynomial $p: \mathbb{R}^{n} \longrightarrow \mathbb{R}^{m}$ and a polynomial $q: \mathbb{R}^{n} \longrightarrow \mathbb{R}$ such that $q^{-1}(0) \cap U=\emptyset$ and $f=p / q$ on $V \cap U$. A (real) algebraic set $V$ is a real algebraic subset of some $\mathbb{R}^{n}$. $V$ shall always be considered equipped with the Euclidean topology inherited from $\mathbb{R}^{n}$. Now let $V$ and $W$ be two nonsingular algebraic sets and let $f: V \longrightarrow W$ be a regular rational map. If $f$ is a diffeomorphism when $V$ and $W$ are considered as smooth manifolds, then $f$ is said to be a rational diffeomorphism. Let $M \subset \mathbb{R}^{n}$ and let $\bar{M}$ be the Zariski closure of $M$ in $\mathbb{R}^{n}$. We say that $M$ is a compact strictly Nash submanifold of $\mathbb{R}^{n}$ if $\bar{M}$ is compact and $M$ is a union of certain nonsingular connected components of $\bar{M}$. Obviously a compact nonsingular algebraic subset of $\mathbb{R}^{n}$ is a compact strictly Nash submanifold of $\mathbb{R}^{n}$.

Let $N$ and $M$ be two smooth manifolds. We denote by $C^{\infty}(N, M)$ the set of all smooth maps from $N$ to $M$ equipped with the $C^{\infty}$-topology.

Let $g: N \longrightarrow M$ and $h: N \longrightarrow M$ be two smooth maps. We say that $g$ is homotopic to $h$ if there exists a smooth map $H: N \times[0,1] \longrightarrow M$ such that $H(x, 0)=g(x)$ and $H(x, 1)=h(x)$ for each $x \in N$.

Theorem 1. For each compact smooth manifold $W$ (respectively compact real analytic or Nash submanifold $W$ of $\mathbb{R}^{m}$ ) containing at least two points there exist a compact nonsingular algebraic set $Z$ and a smooth map (resp. real analytic or Nash map) $g: Z \longrightarrow W$ such that, for every rational diffeomorphism $r: Z^{\prime} \longrightarrow Z$ where $Z^{\prime}$ is a compact nonsingular algebraic set and for every diffeomorphism $s: W^{\prime} \longrightarrow W$ where $W^{\prime}$ is a compact Nash submanifold of some $\mathbb{R}^{k}$, we may fix a neighborhood $\mathcal{U}$ of $s^{-1} \circ g \circ r$ in $C^{\infty}\left(Z^{\prime}, W^{\prime}\right)$ which does not contain any regular rational map. Furthermore if $W^{\prime}$ is a compact strictly Nash submanifold of some $\mathbb{R}^{k}$, then $s^{-1} \circ g \circ r$ is not homotopic to any regular rational map.

Let $V$ be a nonsingular algebraic set, let $W$ be a subset of $V$ and let $U$ be an open neighborhood of $W$ in $V$. A regular rational map $\varrho: U \longrightarrow W$ is a rational retraction of $U$ on $W$ if $\varrho(x)=x$ for each $x \in W$. We say that $W$ has an algebraic tubular neighborhood in $V$ if there is a rational retraction of some open neighborhood of $W$ in $V$ on $W$. 
Piecing together the Weierstrass approximation theorem with Theorem 1 we obtain, at once, the following result.

Theorem 2. Let $W$ be a compact Nash submanifold of $\mathbb{R}^{m}$ containing at least two points. Then $W$ does not have any algebraic tubular neighborhood in $\mathbb{R}^{m}$. In particular if $U$ is a tubular neighborhood of $W$ with submersive smooth retraction $\varrho: U \longrightarrow W$ (for example the closest point map), then $\varrho$ is never a regular rational map.

This result was expected. Let us explain the reasons.

Let $W$ be a compact nonsingular algebraic set of $\mathbb{R}^{m}$ containing at least two points. Thanks to Theorem 1 there exists a compact nonsingular algebraic set $Z$ such that the set $\mathcal{R}(Z, W)$ of all regular rational maps from $Z$ to $W$ is not dense in $C^{\infty}(Z, W)$, in other words a Weierstrass-type approximation theorem for smooth maps from $Z$ to $W$ is false. Notwithstanding the many existing generalizations of the Weierstrass approximation theorem (see [9], [10, 11, 13, [15, 16], 18, 20] and section 13.3 in [8]), in general, there are well-known obstructions to the possibility of approximating a smooth map from a compact nonsingular algebraic set to $W$ by regular rational maps. In view of the classical Weierstrass approximation theorem, these algebraic obstructions prevent the existence of algebraic tubular neighborhoods of $W$ in $\mathbb{R}^{m}$.

Let us recall the main above-mentioned obstructions. We suppose that there exists an algebraic tubular neighborhood of $W$ in $\mathbb{R}^{m}$. Using the Steenrod representability theorem [27], Tognoli's theorem (see Teorema 3 of [28] or Theorem 14.1.10 of [8]) and the existence of an algebraic tubular neighborhood of $W$ in $\mathbb{R}^{m}$, it is easy to see that $W$ has totally algebraic homology, namely $H_{k}(W, \mathbb{Z} / 2 \mathbb{Z})=H_{k}^{\text {alg }}(W, \mathbb{Z} / 2 \mathbb{Z})$ for every integer $k$. However in literature there exists a large quantity of examples of compact nonsingular algebraic subsets $W$ of some $\mathbb{R}^{m}$ without totally algebraic homology and hence without algebraic tubular neighborhoods in $\mathbb{R}^{m}$ (see [4], [5], 7], [11, [14], 17], 19], [22, [23], [24], 26] and section 11.3 in [8]). When $W$ is not connected, the obstruction to the existence of algebraic tubular neighborhoods of $W$ in $\mathbb{R}^{m}$ is obtained in Théorème 2 of 29 making use of a property of the degree of certain regular rational maps (see Lemma 1.2 of [6], see also section 5 of [4]). Finally, Theorem 2 says that the unique compact Nash submanifold $W$ of some $\mathbb{R}^{m}$, which has an algebraic tubular neighborhood in $\mathbb{R}^{m}$, is the single point.

In Section 4 we will present two applications of our results and in Section 5 we will briefly investigate the problem of when there exists a "local algebraic tubular neighborhood".

\section{VARIANTS OF SOME REMARKABLE RESULTS}

Let $P$ be a compact smooth manifold, let $M$ be a compact nonsingular algebraic set and let $q: P \longrightarrow M$ be a smooth map. The unoriented bordism class $\beta$ of $q$ is algebraic if $\beta$ is also represented by a regular rational map $q^{\prime}: P^{\prime} \longrightarrow M$ where $P^{\prime}$ is a compact nonsingular algebraic set.

We are interested in the following version of the Benedetti-Dedò-Teichner theorem.

Theorem 3. For each $d \geq 6$ there exist a d-dimensional compact connected nonsingular algebraic set $M, \bar{a}(d-2)$-dimensional compact connected nonsingular algebraic set $N$ and a smooth map $f: N \longrightarrow M$ such that, for every diffeomorphism 
$a: M^{\prime} \longrightarrow M$ where $M^{\prime}$ is a nonsingular algebraic set, the unoriented bordism class of $a^{-1} \circ f$ is not algebraic. In particular the following assertion is false: there exist diffeomorphisms $b: N^{\prime} \longrightarrow N$ and $c: M^{\prime} \longrightarrow M$ where $N^{\prime}$ and $M^{\prime}$ are nonsingular algebraic sets and a regular rational map $f^{\prime}: N^{\prime} \longrightarrow M^{\prime}$ such that $f^{\prime}$ is arbitrarily close to $c^{-1} \circ f \circ b$ in $C^{\infty}\left(N^{\prime}, M^{\prime}\right)$.

Proof. It suffices to repeat the proof of Theorem 2 in [5] (page 150) also using Tognoli's theorem mentioned above.

In the paper [2], Akbulut and King give a complete topological classification of all real algebraic sets with isolated singularities. One of the basic tools used to obtain this classification is a result which allows us to approximate a smooth map $f$ between compact nonsingular algebraic sets by regular rational maps after slightly modifying the source of $f$. More precisely we are referring to Lemma 2.4 of [2] (see also Proposition 2 of [3]). We need such a lemma in the following form.

Lemma 4. Let $N$ be a compact Nash submanifold of $\mathbb{R}^{n}$, let $M$ be a compact Nash submanifold of $\mathbb{R}^{m}$ and let $f: N \longrightarrow M$ be a smooth map. Then there are a compact $N$ ash submanifold $Z$ of $N \times \mathbb{R}^{m}$, an open subset $Z_{0}$ of $Z$ and a regular rational map $P: Z \longrightarrow M$ such that: if $\pi: N \times \mathbb{R}^{m} \longrightarrow N$ is the natural projection, then $\left.\pi\right|_{Z_{0}}: Z_{0} \longrightarrow N$ is a Nash isomorphism and $\left.P\right|_{Z_{0}}$ is arbitrarily close to $\left.f \circ \pi\right|_{Z_{0}}$ in $C^{\infty}\left(Z_{0}, M\right)$. Moreover if both $N$ and $M$ are compact nonsingular algebraic sets, then $Z$ is a compact nonsingular algebraic set also.

Proof. We give a proof. By the Weierstrass approximation theorem, we may fix a polynomial map $g: \mathbb{R}^{n} \longrightarrow \mathbb{R}^{m}$ such that $\left.g\right|_{N}$ is arbitrarily close to $f$ in $C^{\infty}\left(N, \mathbb{R}^{m}\right)$. Let $U$ be a tubular neighborhood of $M$ in $\mathbb{R}^{m}$ and let $\varrho: U \longrightarrow M$ be the closest point map. We may suppose $g$ so close to $f$ on $N$ that $g(N) \subset U$ and the distance from each point of $g(N)$ to $M$ is less than $\mu$ for some $\mu>0$.

Let $\mathcal{M}:=\left\{(y, v) \in M \times \mathbb{R}^{m} \mid v\right.$ is orthogonal to $\left.T_{y}(M)\right\}$ be the embedded normal bundle of $M$ in $\mathbb{R}^{m}$, let $\gamma: N \times \mathbb{R}^{m} \longrightarrow \mathbb{R}^{m} \times \mathbb{R}^{m}$ be the regular rational map defined by $\gamma(x, v):=(g(x)+v, v)$ and let $Z:=\gamma^{-1}(\mathcal{M})$. Let us briefly study the set $Z$. Obviously $Z$ is a Nash subset of $N \times \mathbb{R}^{m}$. $Z$ is also compact, in fact it is bounded in $N \times \mathbb{R}^{m}: N$ and $M$ are compact so $g(N)$ and $M$ are contained in a ball of $\mathbb{R}^{m}$ centered in the origin with a certain radious $R$, in particular if $(x, v) \in Z$, then $|v| \leq|g(x)+v|+|g(x)|<2 R$ where $|v|$ is the usual norm of $v$ in $\mathbb{R}^{m}$. Now we show that $\gamma$ is transverse to $\mathcal{M}$ so $Z$ will be a compact Nash submanifold of $N \times \mathbb{R}^{m}$ having dimension equal to $(\operatorname{dim} N+m)+m-2 m=\operatorname{dim} N$. Let $(x, v) \in Z$ and let $y:=g(x)+v \in M$. By definition of $\mathcal{M}$ we have that $T_{(y, v)}(\mathcal{M})=T_{y}(M) \times T_{y}^{\perp}(M) \subset \mathbb{R}^{m} \times \mathbb{R}^{m}$ where $T_{y}^{\perp}(M)$ is the vectorial subspace of $\mathbb{R}^{m}$ orthogonal to $T_{y}(M)$. It is easy to verify that $d \gamma_{(x, v)}\left(T_{(x, v)}\left(N \times \mathbb{R}^{m}\right)\right)$ contains the diagonal $\Delta_{m}$ of $\mathbb{R}^{m} \times \mathbb{R}^{m}$ and $\Delta_{m} \cap\left(T_{y}(M) \times T_{y}^{\perp}(M)\right)=\{(0,0)\}$ so $d \gamma_{(x, v)}\left(T_{(x, v)}\left(N \times \mathbb{R}^{m}\right)\right)+T_{(y, v)}(\mathcal{M})=\mathbb{R}^{m} \times \mathbb{R}^{m}$ as desired. Now let $w: N \longrightarrow M$ be the smooth map defined by $\varrho \circ g$ and let $P: Z \longrightarrow M$ be the regular rational map defined by $P(x, v):=g(x)+v$. The open subset $Z_{0}:=\{(x, v) \in Z|| v \mid<\mu\}$ of $Z$ is the graph of the smooth map $(w-g): N \longrightarrow \mathbb{R}^{m}$, hence $\left.\pi\right|_{Z_{0}}: Z_{0} \longrightarrow N$ is a Nash isomorphism. We observe that $P \circ\left(\left.\pi\right|_{Z_{0}}\right)^{-1}(x)=P(x, w(x)-g(x))=w(x)$ for each $x \in N$ so $\left.P\right|_{Z_{0}}=\left.w \circ \pi\right|_{Z_{0}}$. If $g$ is close to $f$, then $w$ is close to $g$ and so $w$ is close to $f$. In particular $\left.P\right|_{Z_{0}}=\left.w \circ \pi\right|_{Z_{0}}$ is close to $\left.f \circ \pi\right|_{Z_{0}}$ in $C^{\infty}\left(Z_{0}, M\right)$ as we want. 
We shall also need a Nash version of Theorem 4 of [3].

Theorem 5. Let $N$ be a compact nonsingular algebraic set, let $M$ be a compact strictly Nash submanifold of $\mathbb{R}^{m}$ and let $f: N \longrightarrow M$ be a smooth map which is homotopic to a regular rational map. Then there are a compact nonsingular algebraic set $N^{\prime}$, a regular rational map $R: N^{\prime} \longrightarrow M$ and a rational diffeomorphism $\varphi: N^{\prime} \longrightarrow N$ such that $R$ is arbitrarily close to $f \circ \varphi$ in $C^{\infty}\left(N^{\prime}, M\right)$.

Proof. Let $\bar{M}$ be the Zariski closure of $M$ in $\mathbb{R}^{m}$. By definition, $\bar{M}$ is compact and $M$ is a union of some nonsingular components of $\bar{M}$. Using Hironaka's resolution theorem, we may suppose $\bar{M}$ to be nonsingular and hence we may apply Theorem 4 of [3] to $f: N \longrightarrow \bar{M}$, completing the proof.

\section{Proof of the theorems}

Proof of Theorem 1 .

First step. Let $W$ be a compact smooth manifold containing at least two distinct points $w$ and $y$. Let $f: N \longrightarrow M$ be as in Theorem 3 . Suppose $N \subset \mathbb{R}^{n}$ and $M \subset$ $\mathbb{R}^{m}$. By Tognoli's theorem there exist a compact nonsingular algebraic set $\bar{W}$ and a diffeomorphism $\psi: \bar{W} \longrightarrow W$. Let $\pi_{N}: N \times \bar{W} \longrightarrow N$ and $\pi_{\bar{W}}: N \times \bar{W} \longrightarrow \bar{W}$ be the natural projections and let $F: N \times \bar{W} \longrightarrow M$ be the smooth map defined by $f \circ \pi_{N}$. Applying Lemma 4 to $F$ we obtain a compact nonsingular algebraic subset $Z$ of $(N \times \bar{W}) \times \mathbb{R}^{m}$, an open subset $Z_{0}$ of $Z$ and a regular rational map $P: Z \longrightarrow M$ such that if $\pi:(N \times \bar{W}) \times \mathbb{R}^{m} \longrightarrow N \times \bar{W}$ is the natural projection, then $\left.\pi\right|_{Z_{0}}: Z_{0} \longrightarrow N \times \bar{W}$ is a Nash isomorphism and $\left.P\right|_{Z_{0}}$ is arbitrarily close to $\left.F \circ \pi\right|_{Z_{0}}$ in $C^{\infty}\left(Z_{0}, M\right)$. In particular $Z$ is the disjoint union of $Z_{0}$ and a compact subset $Q$ of $(N \times \bar{W}) \times \mathbb{R}^{m}$. Let us define the map $g: Z \longrightarrow W$ as follows: $g:=\left.\psi \circ \pi_{\bar{W}} \circ \pi\right|_{Z_{0}}$ on $Z_{0}$ and $g(z):=y$ if $z \in Q$. The map $g$ is a smooth map and, since $\pi_{\bar{W}}$ is transverse to the point $\left\{\psi^{-1}(w)\right\}, g$ is transverse to $\{w\}$ also.

Second step. Let $r: Z^{\prime} \longrightarrow Z$ and $s: W^{\prime} \longrightarrow W$ be as in the statement of the theorem. Let $w^{\prime}:=s^{-1}(w)$ and define $g^{\prime}: Z^{\prime} \longrightarrow W^{\prime}$ by $s^{-1} \circ g \circ r$. By [21] we may choose $w \in W$ in such a way that $w^{\prime}$ is a nonsingular point of the Zariski closure of $W^{\prime}$. The map $g^{\prime}$ is transverse to $\left\{w^{\prime}\right\}$ so

$$
N^{\prime}:=\left(g^{\prime}\right)^{-1}\left(w^{\prime}\right)=r^{-1}\left(\left.\pi\right|_{Z_{0}}\right)^{-1}(N \times\{w\})
$$

is a smooth compact submanifold of $Z^{\prime}$. Suppose $g^{\prime}$ is approximable by regular rational maps in $C^{\infty}\left(Z^{\prime}, W^{\prime}\right)$ and pick a regular rational map $g^{\prime \prime}: Z^{\prime} \longrightarrow W^{\prime}$ close to $g^{\prime}$. If $g^{\prime \prime}$ is sufficiently close to $g^{\prime}$, then $g^{\prime \prime}$ is again transverse to $w^{\prime}$, $N^{\prime \prime}:=\left(g^{\prime \prime}\right)^{-1}\left(w^{\prime}\right)$ is a compact nonsingular algebraic subset of $Z^{\prime}$ and there exists a diffeomorphism $H: Z^{\prime} \longrightarrow Z^{\prime}$ arbitrarily close to the identity such that $H\left(N^{\prime \prime}\right)=$ $N^{\prime}$ (see Theorem 20.2 of [1]). Let $h: N^{\prime \prime} \longrightarrow N^{\prime}$ be the diffeomorphism defined by $h:=\left.H\right|_{N^{\prime \prime}}$, let $b: N^{\prime \prime} \longrightarrow N$ be the diffeomorphism defined by $b:=\left.\pi_{N} \circ \pi\right|_{Z_{0}} \circ r \circ h$ and let $f^{\prime \prime}: N^{\prime \prime} \longrightarrow M$ be the regular rational map $\left.P \circ r\right|_{N^{\prime \prime}}$. Since $P$ can be chosen arbitrarily close to $F \circ \pi$ on $Z_{0}$, it follows that $f^{\prime \prime}$ is as close to $F \circ \pi \circ r \circ h=$ $f \circ \pi_{N} \circ \pi \circ r \circ h=f \circ b$ as we want. This is impossible thanks to the last part of Theorem 3. It follows that $g^{\prime}$ cannot be approximated by regular rational maps.

Third step. Let us prove that if $W^{\prime}$ is strictly Nash, then $g^{\prime}$ is not homotopic to any regular rational map. Suppose on the contrary that $g^{\prime}$ is homotopic to a regular rational map. By Theorem 5$]$ applied to $g^{\prime}$ we have a compact nonsingular algebraic set $Z^{\prime \prime}$, a regular rational map $R: Z^{\prime \prime} \longrightarrow W^{\prime}$ and a rational diffeomorphism $\varphi$ : $Z^{\prime \prime} \longrightarrow Z^{\prime}$ such that $R$ is arbitrarily close to $g^{\prime} \circ \varphi$ in $C^{\infty}\left(Z^{\prime \prime}, W^{\prime}\right)$. If $R$ is sufficiently 
close to $g^{\prime} \circ \varphi$, then $R$ is transverse to $w^{\prime}$ (because $g^{\prime} \circ \varphi$ is), $N^{\prime \prime \prime}:=R^{-1}\left(w^{\prime}\right)$ is a compact nonsingular algebraic subset of $Z^{\prime \prime}$ and there is a diffeomorphism $H^{\prime}: Z^{\prime \prime} \longrightarrow Z^{\prime \prime}$ arbitrarily close to the identity such that $H^{\prime}\left(N^{\prime \prime \prime}\right)=\varphi^{-1}\left(N^{\prime}\right)$ (again use Theorem 20.2 of [1] ). Let $h^{\prime}: N^{\prime \prime \prime} \longrightarrow \varphi^{-1}\left(N^{\prime}\right)$ be the diffeomorphism defined by $h^{\prime}:=\left.H^{\prime}\right|_{N^{\prime \prime \prime}}$, let $b^{\prime}: N^{\prime \prime \prime} \longrightarrow N$ be the diffeomorphism defined by $b^{\prime}:=\left.\pi_{N} \circ \pi\right|_{Z_{0}} \circ r \circ \varphi \circ h^{\prime}$ and let $f^{\prime \prime \prime}: N^{\prime \prime \prime} \longrightarrow M$ be the regular rational map defined as the composition $\left.P \circ r \circ \varphi\right|_{N^{\prime \prime \prime}}$. We have that $f^{\prime \prime \prime}$ is arbitrarily close to $F \circ \pi \circ r \circ \varphi \circ h^{\prime}=f \circ b^{\prime}$ and so the last part of Theorem 3 is again contradicted.

Final remark. We conclude the proof by making an observation about the first step. If $W$ is a compact real analytic (Nash) submanifold of some $\mathbb{R}^{m}$, then $g$ may be replaced by a real analytic (Nash) map sufficiently close to $g$ (this is always possible because the set of all real analytic (Nash) maps from $Z$ to $W$ is dense in $\left.C^{\infty}(Z, W)\right)$. With few changes the rest of the proof still works.

Remark 6. Theorem 1 cannot be improved. Let us explain in which sense. First, we state Proposition 2.8 of [2] in a particular case.

Proposition 2.8 ([2]). Let $N$ and $M$ be two compact nonsingular algebraic sets and let $f: N \longrightarrow M$ be a smooth map. Suppose $M$ has totally algebraic homology, namely $H_{k}^{\text {alg }}(M, \mathbb{Z} / 2 \mathbb{Z})=H_{k}(M, \mathbb{Z} / 2 \mathbb{Z})$ for each integer $k$. Then there are a compact nonsingular algebraic set $N^{\prime}$, a diffeomorphism $\psi: N^{\prime} \longrightarrow N$ and a regular rational map $P: N^{\prime} \longrightarrow M$ such that $P$ is arbitrarily close to $f \circ \psi$ in $C^{\infty}\left(N^{\prime}, M\right)$.

Let $W$ be a compact nonsingular algebraic set with totally algebraic homology containing at least two points and let $g: Z \longrightarrow W$ be as in Theorem 1 Applying the previous version of Proposition 2.8 of [2] to $g$ we obtain a compact nonsingular algebraic set $Z^{\prime}$, a diffeomorphism $r: Z^{\prime} \longrightarrow Z$ and a regular rational map $f^{\prime}: Z^{\prime} \longrightarrow W$ such that $f^{\prime}$ is arbitrarily close to $g \circ r$ in $C^{\infty}\left(Z^{\prime}, W\right)$. This fact tells us that in the statement of Theorem 1 we cannot replace "for every rational diffeomorphism $r: Z^{\prime} \longrightarrow Z$ " with "for every diffeomorphism $r: Z^{\prime} \longrightarrow Z$ ". Furthermore, it is well-known that with such choice of $W$ the unoriented bordism group of $W$ is algebraic. In this way, Theorem 1 establishes a clear distinction between the property of a smooth map $f$ to represent an algebraic unoriented bordism class and the property of the map $f$ to be homotopic to a regular rational map.

This remark may also be seen from the point of view of Proposition 2.8 of [2] saying that in such a proposition the diffeomorphism $\psi: N^{\prime} \longrightarrow N$ in general is not a regular rational map.

Proof of Theorem 2. Let $W$ be a compact Nash submanifold of $\mathbb{R}^{m}$ containing at least two points and let $g: Z \longrightarrow W$ be as in the statement of Theorem 11. Consider $g$ as a Nash map from $Z$ to $\mathbb{R}^{m}$. Let us suppose that there exist an open neighborhood $U$ of $W$ in $\mathbb{R}^{m}$ and a rational retraction $\varrho: U \longrightarrow W$ on $W$. By the Weierstrass approximation theorem we may fix a polynomial map $P: Z \longrightarrow \mathbb{R}^{m}$ arbitrarily close to $g$ in $C^{\infty}\left(Z, \mathbb{R}^{m}\right)$. If $P$ is sufficiently close to $g$, then $P(Z) \subset U$ and the regular rational map $\varrho \circ P: Z \longrightarrow W$ is close to $g$ in $C^{\infty}(Z, W)$ as we want. This contradicts Theorem 1 .

\section{Some applications}

In this section we will present two applications of our results. The first concerns an obstruction to improve the Artin-Mazur Theorem and the second regards an 
obstruction to embed birationally in $\mathbb{R}^{e}$ certain open subsets of an ample class of $e$-dimensional algebraic fiber bundles.

Let us recall the Artin-Mazur Theorem (see Theorem 8.4.4 of [8]).

Artin-Mazur Theorem. Let $Y$ be a d-dimensional connected Nash submanifold of $\mathbb{R}^{p}$ and let $f: Y \longrightarrow \mathbb{R}^{m}$ be a Nash map. There exists a d-dimensional nonsingular irreducible algebraic subset $V$ of $\mathbb{R}^{p} \times \mathbb{R}^{k}$ for some integer $k$, an open semialgebraic subset $Y^{\prime}$ of $V$ and a polynomial map $P: V \longrightarrow \mathbb{R}^{m}$ such that if $\pi: \mathbb{R}^{p} \times \mathbb{R}^{k} \longrightarrow \mathbb{R}^{p}$ is the natural projection, then $\left.\pi\right|_{Y^{\prime}}: Y^{\prime} \longrightarrow Y$ is a Nash isomorphism and $P=f \circ \pi$ on $Y^{\prime}$.

A question arises: Can we take $Y^{\prime}=V$ ?

The next proposition gives a negative answer. In particular for each $m$ there are Nash maps $f$ from compact connected Nash submanifolds $Y$ to $\mathbb{R}^{m}$ such that if $Y^{\prime}$ and $V$ satisfy the Artin-Mazur Theorem applied to $f$, then $Y^{\prime}$ is different from $V$.

Proposition 7. For each non-void compact connected Nash submanifold $W$ of $\mathbb{R}^{m}$ there are a compact connected Nash submanifold $Y$ of some $\mathbb{R}^{p}$ and a Nash map $\eta: Y \longrightarrow W$ such that the following assertion is not true: there exist a compact nonsingular algebraic set $Y^{\prime}$, a rational diffeomorphism $\varphi: Y^{\prime} \longrightarrow Y$ and a regular rational map $R: Y^{\prime} \longrightarrow W$ such that $R=\eta \circ \varphi$. In particular if $V, Y^{\prime}$ and $P$ satisfy the conclusion of the Artin-Mazur Theorem applied to $\eta$, then $Y^{\prime} \neq V$.

Proof. The proof is similar to the one of Theorem 1 Let $f: N \longrightarrow M$ be as in Theorem [3. Repeat the first step of the proof of Theorem 1 with $\bar{W}:=W$. We obtain a compact Nash submanifold $Z_{0}$, a Nash isomorphism $\pi: Z_{0} \longrightarrow N \times W$ and a regular rational map $P: Z_{0} \longrightarrow M$ such that $P$ is arbitrarily close to $f \circ \pi_{N} \circ \pi$ in $C^{\infty}\left(Z_{0}, M\right)$. Define $Y:=Z_{0}$ and $\eta: Y \longrightarrow W$ by $\pi_{W} \circ \pi$. Since $N$ and $W$ are connected, it follows that $Y$ is also connected. Suppose that there exist a compact nonsingular algebraic set $Y^{\prime}$, a rational diffeomorphism $\varphi: Y^{\prime} \longrightarrow Y$ and a regular rational map $R: Y^{\prime} \longrightarrow W$ such that $R=\eta \circ \varphi$. Fix $w \in W$ as in the second step of the proof of Theorem $\Pi$ with $s$ equal to the identity on $W$. Observe that $\eta$ is transverse to $\{w\}$ so $R$ is transverse to $\{w\}$ also. Let $N^{\prime}:=R^{-1}(w)$. $N^{\prime}$ is a compact nonsingular algebraic subset of $Y^{\prime}$. Let $b: N^{\prime} \longrightarrow N$ be the diffeomorphism $\left.\pi_{N} \circ \pi \circ \varphi\right|_{N^{\prime}}$ and let $f^{\prime}: N^{\prime} \longrightarrow M$ be the regular rational map $\left.P \circ \varphi\right|_{N^{\prime}}$. Since $P$ may be chosen arbitrarily close to $f \circ \pi_{N} \circ \pi$ in $C^{\infty}\left(Z_{0}, M\right)$, we have that $f^{\prime}$ is close to $f \circ b$ in $C^{\infty}\left(N^{\prime}, M\right)$ as we want. This contradicts Theorem 3

If $W$ consists of a single point, then the above proof gives the following result: for each $d \geq 4$, there is a d-dimensional compact connected Nash submanifold $Y$ of some $\mathbb{R}^{p}$ such that if $Y^{\prime}$ is an algebraic model of $Y$ and $\psi: Y^{\prime} \longrightarrow Y$ is a diffeomorphism, then $\psi$ is never a regular rational map.

Let us proceed with another application.

Let $E$ be an $e$-dimensional abstract algebraic manifold and let $U$ be an open subset of $E$. We say that $U$ can be birationally embedded in $\mathbb{R}^{e}$ if there exist an open subset $U^{\prime}$ of $\mathbb{R}^{e}$ and a regular birational isomorphism from $U$ to $U^{\prime}$, namely a regular rational map $\varphi: U \longrightarrow U^{\prime}$ having a regular rational inverse $\varphi^{-1}: U^{\prime} \longrightarrow U$.

Let $W$ be a nonsingular algebraic set. A topological fiber bundle $\pi: E \longrightarrow W$ over $W$ is algebraic if $E$ is an algebraic abstract manifold and $\pi$ is a submersive regular rational map. 
Proposition 8. Let $W$ be a compact nonsingular algebraic subset of $\mathbb{R}^{m}$ containing at least two points, let $\pi: E \longrightarrow W$ be an algebraic fiber bundle over $W$, let $\sigma: W \longrightarrow E$ be a regular rational section of $\pi$ and let $U$ be an open neighborhood of $\sigma(W)$ in $E$. If $\operatorname{dim}(E)=e$, then $U$ cannot be birationally embedded in $\mathbb{R}^{e}$.

In particular the following is true.

Let $E:=\left\{(x, v) \in W \times \mathbb{R}^{m} \mid v\right.$ is orthogonal to $\left.T_{x}(W)\right\}$, let $\pi: E \longrightarrow W$ be the normal bundle of $W$ in $\mathbb{R}^{m}$ and let $\theta: E \longrightarrow \mathbb{R}^{m}$ be the polynomial map defined by $\theta(x, v):=x+v$. By the Tubular Neighborhood Theorem there are an open neighborhood $U_{0}$ of the zero section of $\pi$ in $E$ and an open neighborhood $U$ of $W$ in $\mathbb{R}^{m}$ such that $\left.\theta\right|_{U_{0}}: U_{0} \longrightarrow U$ is a rational diffeomorphism. Then $\left(\left.\theta\right|_{U_{0}}\right)^{-1}$ is never a regular rational map.

Proof. If there would exist a birational map $\varphi: U \longrightarrow U^{\prime}$ where $U^{\prime}$ is an open subset of $\mathbb{R}^{e}$, then the map $\varrho: U^{\prime} \longrightarrow \varphi \circ \sigma(W)$ defined by $\varphi \circ \sigma \circ \pi \circ \varphi^{-1}$ would be a rational retraction from $U^{\prime}$ on the compact nonsingular algebraic subset $\varphi \circ \sigma(W)$ of $\mathbb{R}^{e}$. Now $\varphi \circ \sigma(W)$ contains at least two points, so Theorem 2 is contradicted.

For other algebraic obstructions of the same kind we refer to [6] and [7.

\section{Final remarks about rational Retractions}

In Theorem 2 we proved that the problem regarding the existence of algebraic tubular neighborhoods of a compact nonsingular algebraic subset $W$ of $\mathbb{R}^{m}$ is solvable only when $W$ is a single point. In this section we will study briefly the existence of local rational retractions on $W$.

First, let us give a definition. Let $V$ be a nonsingular algebraic set, let $W$ be a subset of $V$ and let $p$ be a point of $W$. We say that $W$ has a rational retraction locally at $p$ in $V$ if there exist an open neighborhood $U$ of $p$ in $V$ and a rational retraction of $U$ on $U \cap W$. The following is a simple result.

Proposition 9. Let $R$ be a polynomial of $\mathbb{R}\left[x_{1}, \ldots, x_{n}\right]$ of degree less than or equal to 2 , let $V:=R^{-1}(0)$ and let $p \in V$ such that the differential $d R_{p}$ of $R$ at $p$ is not null. Then $V$ has a rational retraction locally at $p$ in $\mathbb{R}^{n}$.

Furthermore the following is true. Let $q$ be a point of $V$. Suppose the degree of $R$ to be exactly 2 and write $R(x)=R_{1}(x-q)+R_{2}(x-q)$ where each $R_{k}$ is a homogeneous polynomial of $\mathbb{R}\left[x_{1}, \ldots, x_{n}\right]$ of degree $k$. Let $C_{q}$ be the cone of $\mathbb{R}^{n}$ with vertex $q$ defined by $C_{q}:=\left\{x \in \mathbb{R}^{n} \mid R_{1}(x-q) \cdot R_{2}(x-q)=0\right\}$. If $V$ is not contained in $C_{q}$, then the map $\varrho_{p}: \mathbb{R}^{n} \backslash C_{q} \longrightarrow V \backslash C_{q}$ defined by

$$
\varrho_{p}(x):=q-\frac{R_{1}(x-q)}{R_{2}(x-q)} \cdot(x-q)
$$

is a rational retraction of $\mathbb{R}^{n} \backslash C_{q}$ on $V \backslash C_{q}$.

Proof. Using an affinity if needed we may suppose that $p$ is equal to the origin 0 of $\mathbb{R}^{n}$ and the Zariski tangent space of $V$ at 0 is $\left\{x \in \mathbb{R}^{n} \mid x_{n}=0\right\}$ which we identify with $\mathbb{R}^{n-1}$. If $V$ coincides with $\mathbb{R}^{n-1}$ locally at 0 , then the natural projection $\pi: \mathbb{R}^{n} \longrightarrow \mathbb{R}^{n-1}$, suitably restricted locally at 0 , is a desired rational retraction. Now suppose $V$ to be different from $\mathbb{R}^{n-1}$ locally at 0 . By this hypothesis we may pick a point $q \in V \backslash\{0\}$ arbitrarily close to 0 such that the straight line $r$ containing $q$ and 0 does not lie completely in $V$ (otherwise $V=\mathbb{R}^{n-1}$ locally at 0 ). We write $R(x)=R_{1}(x-q)+R_{2}(x-q)$ and define $C_{q}$ and $\varrho_{q}$ as in the second 
part of the statement. The map $\varrho_{q}$ is a rational retraction of $\mathbb{R}^{n} \backslash C_{q}$ on $V \backslash C_{q}$. In fact, $R\left(\varrho_{q}(x)\right)=0$ and $\varrho_{q}(x) \notin C_{q}$ for each $x \in \mathbb{R}^{n} \backslash C_{q}$; moreover $\varrho_{q}(x)=x$ for each $x \in V \backslash C_{q}$. It remains to be proved that $0 \notin C_{q}$ so $\varrho_{q}$ will turn out to be a desired rational retraction. The straight line $r$ is the image of $\gamma: \mathbb{R} \longrightarrow \mathbb{R}^{n}$ defined by $\gamma(t):=q-t q$. By assumption $r \not \subset V$ so the polynomial equation $0=R(\gamma(t))=t^{2} R_{2}(-q)+t R_{1}(-q)$ must have a finite number of solutions in $t$ which are $t=0$ and $t=1$. It follows that $R_{2}(-q)=-R_{1}(-q) \neq 0$ and so $0 \notin C_{q}$.

In the previous proposition if $V$ is a $(n-1)$-sphere $S$ of $\mathbb{R}^{n}$ and $R$ is the standard quadratic polynomial equation of $S$ in $\mathbb{R}^{n}$, then it is easy to verify that, for each $q \in S$, the cone $C_{q}$ is exactly the tangent space of $S$ at $q$. In particular $S \cap C_{q}=\{q\}$ and so $S \backslash\{q\}$ has an algebraic tubular neighborhood in $\mathbb{R}^{n}$.

Let us make another observation about Proposition 9 Let $V$ be the vanishing set of a polynomial $R \in \mathbb{R}\left[x_{1}, \ldots, x_{n}\right]$ of degree $d \geq 2$ and let $q \in V$ such that $R$ can be written as follows: $R(x)=R_{d-1}(x-q)+R_{d}(x-q)$ where each $R_{k}$ is a homogeneous polynomial of $\mathbb{R}\left[x_{1}, \ldots, x_{n}\right]$ of degree $k$. Under these hypotheses the second part of Proposition 9 remains true with $C_{q}$ and $\varrho_{q}$ defined as above replacing $R_{1}$ with $R_{d-1}$ and $R_{2}$ with $R_{d}$.

Let us conclude with an obstruction result.

A 1-dimensional nonsingular real algebraic subset of $\mathbb{R}^{n}$ (respectively $\mathbb{R P}^{n}$ ) is called an affine (projective) real algebraic curve of $\mathbb{R}^{n}$ (resp. $\mathbb{R P}^{n}$ ). Let $C$ be an affine (projective) real irreducible algebraic curve of $\mathbb{R}^{n}$ (resp. $\mathbb{R P}^{n}$ ). By Hironaka's resolution of singularities theorem there exists a projective complex irreducible curve $C_{\mathbb{C}}$ defined over $\mathbb{R}$ such that $C$ is regular birational isomorphic to a Zariski open subset of the set of all real points $C_{\mathbb{C}}(\mathbb{R})$ of $C_{\mathbb{C}}$ where $C_{\mathbb{C}}(\mathbb{R})$ is viewed as a projective real algebraic curve. Since $C_{\mathbb{C}}$ is unique up to birational isomorphism over $\mathbb{R}$, we may define the genus $g(C)$ of $C$ as the genus of $C_{\mathbb{C}}$. Moreover we recall that every regular rational map $r: C \longrightarrow D$ between affine (projective) real irreducible algebraic curves of $\mathbb{R}^{n}$ (resp. $\mathbb{R P}^{n}$ ) admits a unique complex regular rational extension $r_{\mathbb{C}}$ from $C_{\mathbb{C}}$ to $D_{\mathbb{C}}$.

Proposition 10. Let $C$ be an affine (projective) real irreducible algebraic curve of $\mathbb{R}^{n}$ (resp. $\mathbb{R P}^{n}$ ) such that $g(C)>0$. Fix a point $p$ of $C$. Then $C$ does not have any rational retraction locally at $p$ in $\mathbb{R}^{n}\left(\right.$ resp. $\left.\mathbb{R} \mathbb{P}^{n}\right)$.

Proof. Since the problem is local, it suffices to consider the affine case. Assume that there is a rational retraction $\varrho$ from an open neighborhood $U$ of $p$ on $C$. Observe that, by hypotheses, it follows that $n \geq 2$. After an affinity of $\mathbb{R}^{n}$, we may suppose that $p$ is equal to the origin 0 of $\mathbb{R}^{n}$ and the Zariski tangent space of $C$ at 0 is $\mathbb{R}_{x_{1}}:=\left\{x \in \mathbb{R}^{n} \mid x_{2}=\ldots=x_{n}=0\right\}$. Let $\mathbb{R}_{x_{1} x_{2}}:=\left\{x \in \mathbb{R}^{n} \mid x_{3}=\ldots=x_{n}=0\right\}$ and let $\pi: \mathbb{R}_{x_{1} x_{2}} \longrightarrow \mathbb{R}_{x_{1}}$ be the natural projection. Since $\varrho(x)=x$ for each $x \in U \cap C$ and $\mathbb{R}_{x_{1}}$ is the Zariski tangent space of $C$ at 0 , it is easy to establish that $\left.\varrho\right|_{U \cap \mathbb{R}_{x_{1}}}: U \cap \mathbb{R}_{x_{1}} \longrightarrow C$ is a local diffeomorphism at 0 . Let $\varepsilon>0$ such that the image under $\pi$ of the circle $S_{\varepsilon}^{1}:=\left\{\left(x_{1}, x_{2}\right) \in \mathbb{R}_{x_{1} x_{2}} \mid x_{1}^{2}+x_{2}^{2}=\varepsilon^{2}\right\}$ is contained in $U \cap \mathbb{R}_{x_{1}}$. The regular rational map $r: S_{\varepsilon}^{1} \longrightarrow C$ defined by $r:=\left.\varrho \circ \pi\right|_{S_{\varepsilon}^{1}}$ turns out to be well-defined and nonconstant. Let $r_{\mathbb{C}}:\left(S_{\varepsilon}^{1}\right)_{\mathbb{C}} \longrightarrow C_{\mathbb{C}}$ be the complex extension of $r$. Since $r_{\mathbb{C}}$ is not constant, by the Riemann-Hurwitz formula we have that $g(C) \leq g\left(S_{\varepsilon}^{1}\right)=0$, which contradicts our hypotheses. 
We refer to [15] and [16] for other results derived from techniques similar to the one used in the previous proof. For the problem of the existence of "local Nash tubular neighborhoods" in the complex case we refer to [25].

\section{REFERENCES}

1. R. Abraham and J. Robbin, Transversal mapping and flows, New York, Benjamin (1967). MR 39:2181

2. S. Akbulut and H. C. King, The topology of real algebraic sets with isolated singularities, Ann. of Math. 113 (1981), no. 3, 425-446. MR 83b:58003

3. S. Akbulut and H. C. King, A resolution theorem for homology cycles of real algebraic varieties, Invent. Math. 79 (1985), no. 3, 589-601. MR 86g:14011

4. S. Akbulut and H. C. King, Submanifolds and homology of nonsingular algebraic varieties, Amer. J. Math. 107 (1985), no. 1, 87-98. MR 93d:57051

5. R. Benedetti and M. Dedò, Counterexamples to representing homology classes by real algebraic subvarieties up to homeomorphism, Compositio Math. 53 (1984), no. 2, 143-151. MR 86h:57031

6. R. Benedetti and A. Tognoli, On real algebraic vector bundles, Bull. Sci. Math. 104 (1980), no. 1, 89-112. MR 81e:14009

7. R. Benedetti and A. Tognoli, Remarks and counterexamples in the theory of real algebraic vector bundles and cycles, Géométrie algébrique réelle et formes quadratiques. Lecture Notes in Math. 959, 198-211, Berlin: Springer-Verlag (1982). MR 85a:14018

8. J. Bochnak, M. Coste and M.-F. Roy, Real Algebraic Geometry, Berlin: Springer-Verlag (1998). MR 2000a:14067

9. J. Bochnak and W. Kucharz, Algebraic approximation of mappings into spheres, Michigan Math. J. 34 (1987), no. 1, 119-125. MR 88h:58018

10. J. Bochnak and W. Kucharz, On real algebraic morphisms into even-dimensional spheres, Ann. of Math. 128 (1988), no. 2, 415-433. MR 89k:57060

11. J. Bochnak and W. Kucharz, Algebraic models of smooth manifolds, Invent. Math. 97 (1989), no. 3, 585-611. MR 91b:14076

12. J. Bochnak and W. Kucharz, Algebraic cycles and approximation theorems in real algebraic geometry, Trans. Amer. Math. Soc. 337 (1993), no. 1, 463-472. MR 93g:57033

13. J. Bochnak and W. Kucharz, Elliptic curves and real algebraic morphisms, J. Algebraic Geom. 2 (1993), no. 4, 635-666. MR 94e:14072

14. J. Bochnak and W. Kucharz, Real algebraic hypersurfaces in complex projective varieties, Math. Ann. 301 (1995), no. 2, 381-397. MR 97d:14081

15. J. Bochnak and W. Kucharz, The Weierstrass approximation theorem and a characterization of the unit circle, Proc. Amer. Math. Soc. 127 (1999), no. 6, 1571-1574. MR 99i:14067

16. J. Bochnak and W. Kucharz, The Weierstrass approximation theorem for maps between real algebraic varieties, Math. Ann. 314 (1999), no. 4, 601-612. MR 2001c:14082

17. J. van Hamel, Real algebraic cycles on complex projective varieties, Math. Z. 225 (1997), no. 2, 177-198. MR 98i:14012

18. N. Ivanov, Approximation of smooth manifolds by real algebraic sets, Russian Math. Surveys 37 (1982), no. 1, 1-59. MR 84i:57029

19. W. Kucharz, On homology of real algebraic sets, Invent. Math. 82 (1985), no. 1, 19-25. MR 87b:57025

20. W. Kucharz, Algebraic morphisms into rational real algebraic surfaces, J. Algebraic Geom. 8 (1999), no. 3, 569-579. MR 2000d:14064

21. F. Lazzeri and A. Tognoli, Alcune proprietà degli spazi algebrici, Ann. Scuola Norm. Sup. Pisa 24 (1970), no. 3, 597-632. MR 45:1909

22. F. Mangolte, Cycles algégriques sur les surfaces K3 réelles, Math. Z. 225 (1997), no. 4, 559-576. MR 98f:14046

23. J.-J. Risler, Sur l’homologie des surfaces algébriques réelles, Géométrie algébrique réelle et formes quadratiques. Lecture Notes in Math. 959, 381-385, Berlin: Springer-Verlag (1982). MR 84c:14019

24. R. Silhol, $A$ bound on the order of $H_{n-1}^{(a)}(X, \mathbb{Z} / 2)$ on a real algebraic variety, Géométrie algébrique réelle et formes quadratiques. Lecture Notes in Math. 959, 443-450, Berlin: Springer-Verlag (1982). MR 84e:14024 
25. A. Tancredi and A. Tognoli, On the extension of Nash functions, Math. Ann. 228 (1990), no. 4, 595-604. MR 92c:32011

26. P. Teichner, 6-dimensional manifolds without totally algebraic homology, Proc. Amer. Math. Soc. 123 (1995), no. 9, 2909-2914. MR 95k:57031

27. R. Thom, Quelques propriétés globales des variétiés difféntiables, Comment. Math. Helv. 28 (1954), 17-86. MR 15:890a

28. A. Tognoli, Su una congettura di Nash, Ann. Scuola Norm. Sup. Pisa 27 (1973), no. 3, 167-185. MR 53:434

29. A. Tognoli, Une remarque sur les approximations en géométrie algébrique réelle, $\mathrm{C}$. $\mathrm{R}$. Acad. Sci. Paris S'er. I Math. 296 (1983), no. 17, 745-747. MR 85b:14031

Dipartimento di Matematica, University of Pisa, via Buonarroti 2, 56127 Pisa, Italy

E-mail address: ghiloni@mail.dm.unipi.it 